\title{
SPECTRAL THEORY OF REINHARDT MEASURES
}

\author{
RAÚL E. CURTO AND KEREN YAN
}

Let $\mu$ be a finite positive Borel measure on $\mathbf{C}^{n}(n \geq 1)$, with compact support $K$, let $P^{2}(\mu)$ be the norm closure in $L^{2}(\mu)$ of the algebra of complex polynomials in $z_{1}, \ldots, z_{n}$, and let $M_{z}=\left(M_{z_{1}}, \ldots, M_{z_{n}}\right)$ be the $n$-tuple of multiplication operators by the coordinate functions $z_{1}, \ldots, z_{n}$ acting on $P^{2}(\mu) . M_{z}$ is the universal model for cyclic subnormal $n$-tuples of operators acting on a separable Hilbert space. For $n=1$, the spectral and algebraic properties of $M_{z}$ have been the focus of extensive study (see [Con] for a survey account of the basic results in this area). One important instance, the case $d \mu\left(\mathrm{re}^{i \theta}\right)=d \rho(r) \times \frac{d \theta}{2 \pi}$ (where $\rho$ is a positive Borel measure on $[0,+\infty)$ ), gives rise to the class of subnormal weighted shifts, via Berger's Theorem [Con, III.8.16]. Here, the spectral picture of $M_{z}$ admits a very simple description:

(i) $\sigma\left(M_{z}\right)$, the spectrum of $M_{z}$, equals $D_{\mu}:=\{\lambda \in \mathrm{C}:|\lambda| \leq$ $\sup \{|z|: z \in K\}\}$

(ii) The Fredholm domain of $M_{z}$ is $\mathbf{C} \backslash \partial D_{\mu}$; and

(iii) index $\left(M_{z}-\lambda\right)=-1$ whenever $\lambda \in \operatorname{int}\left(D_{\mu}\right)$.

The circular symmetry of weighted shifts, reflected in the above description, appears in several variables in the notion of Reinhardt set; $F \subseteq \mathbf{C}^{n}$ is Reinhardt if $F=\tau^{-1}(\tau(F))$, where $\tau: \mathbf{C}^{n} \rightarrow \mathbf{R}_{+}^{n}$ is given by $z \rightarrow\left(\left|z_{1}\right|, \ldots,\left|z_{n}\right|\right)$. Correspondingly, a compactly supported positive Borel measure $\mu$ is Reinhardt if it admits a decomposition $d \mu\left(\mathrm{re}^{i \theta}\right)=d \rho(r) \times d \theta /(2 \pi)^{n}$, where $\rho$ is a positive Borel measure on $\mathbf{R}_{+}^{n}$. For instance, volumetric Lebesgue measure on a complete bounded Reinhardt domain $\Omega \subseteq \mathbf{C}^{n}$ is a Reinhardt measure, in which case $P^{2}(\mu)$ is actually $A^{2}(\Omega)$, the Bergman space over $\Omega$.

Received by the editors April 18, 1990.

1980 Mathematics Subject Classification (1985 Revision). Primary 47A10, 47A53, 47B37, 32A07; Secondary 47B20, 32E20, 47B35, 47A50.

The research of the first author was partially supported by NSF Grant MCS880139 and by a University of Iowa Faculty Scholar Award.

The research of the second author was partially supported by NSF Grant DMS 9002969. 
The spectral and $C^{*}$-algebraic properties of $M_{z}$ on $A^{2}(\Omega)$, for $\Omega \subseteq \mathbf{C}^{n}$ Reinhardt or pseudoconvex, have been extensively investigated, as have been those of $M_{z}$ acting on the Hardy spaces over the Shilov boundary of bounded symmetric domains (e.g., [BC, BCK, BCZ, BdeM, Cob, CM, CS, DH, MR, P, Ra, SSU, $\mathrm{U}, \mathrm{V}])$. In this note we announce a complete description of the spectral picture of $M_{z}$ in case $\mu$ is a Reinhardt measure on $\mathbf{C}^{2}$ whose associated weight sequences have limits at infinity in all directions (a notion to be defined later).

To describe our results, we need some notation. Let

$$
V:=\left\{\left(z_{1}, \ldots, z_{n}\right) \in \mathbf{C}^{n}: z_{1} \cdots z_{n}=0\right\} .
$$

Without loss of generality, we can, and shall, assume that $K \subseteq$ $\bar{D}^{n}$ and that $K$ is not contained in $V$, since otherwise $M_{z}$ is unitarily equivalent to the orthogonal direct sum of $n$-tuples of the form $\left(M_{\left(z_{1}, \ldots, z_{k}\right)}, 0, \ldots, 0\right)$. For $\mu$ Reinhardt, the set of bounded point evaluations for $\mu$ is b.p.e. $(\mu):=\left\{\lambda \in \mathbf{C}^{n}: p \rightarrow\right.$ $p(\lambda), p \in \mathbf{C}[z]$, extends boundedly to $\left.P^{2}(\mu)\right\}$. The Taylor spectrum of $M_{z}, \sigma_{T}\left(M_{z}\right)$, is a nonempty compact subset of $\mathbf{C}^{n}$ defined in terms of the exactness of a cochain complex, called the Koszul complex, built from the exterior algebra on $n$ generators and the coordinates $M_{z_{i}}, i=1, \ldots, n$. The Taylor spectrum enjoys most of the usual properties of the spectrum of a single Hilbert space operator, and supports an analytic functional calculus. There is also a notion of Fredholmness and of index for commuting $n$-tuples of operators. (For basic facts on joint spectral systems, the reader is referred to [Cu].) Finally, for a compact subset $F$ of $\mathbf{C}^{n}$, we let $\widehat{F}$ denote the polynomially convex hull of $F$. If $F$ is Reinhardt and $0 \in F$, then $\widehat{F}=$ $\tau^{-1}\{\exp [\text { convex hull }(\log (\tau(F) \backslash V))]\}^{-}$. In particular, if $z \in F$ then the polydisk $\left\{w \in \mathbf{C}^{n}:\left|w_{i}\right| \leq\left|z_{i}\right|, i=1, \ldots, n\right\}$ is contained in $\widehat{F}$.

Theorem 1. Let $\mu$ be a Reinhardt measure on $\mathbf{C}^{n}$. Then

(i) int $\widehat{K} \subseteq$ b. p.e. $(\mu) \subseteq \widehat{K}$;

(ii) $\sigma_{T}\left(M_{z}^{(\mu)}\right)=\widehat{K}$.

To prove Theorem 1(i), we construct a dense-range operator from $P^{2}(\mu)$ to the Hardy space of the $n$-torus, $H^{2}\left(\mathbf{T}^{n}\right)$, and we then use it to pull back the Szegö kernel function from $H^{2}\left(\mathrm{~T}^{n}\right)$ to $P^{2}(\mu)$; part (ii) requires a spectral inclusion $[\mathrm{Cu}$, Theorem 
7.5(ii)] together with the containment b.p.e. $(\mu) \subseteq \sigma_{T}\left(M_{z}\right)$. To discuss our calculation of the Taylor essential spectrum of $M_{z}$, we require some preparations. To begin with, the existence of bounded point evaluations in a neighborhood $\Omega$ of the origin (Theorem 1(i)) gives rise to a kernel function $k(w, z)$ such that $f(z)=\langle f, k(\cdot, z)\rangle$ for all $f \in P^{2}(\mu), z \in \Omega$. Let $\lambda \in K \backslash V$ and let $\varepsilon:=\left(\min _{i}\left|\lambda_{i}\right|\right) / 3$. Use of the Cauchy-Schwarz inequality now yields the following key estimate: There exists a constant $C>0$ such that

$$
\iint_{\bar{D}(0, \varepsilon) \cap K}|f|^{2} d \mu \leq C \iint_{K \backslash \bar{D}(0, \varepsilon)}|f|^{2} d \mu,
$$

for every $f \in P^{2}(\mu)$, where $D(0, \varepsilon)$ is the open polydisk centered at the origin and of multiradius $(\varepsilon, \ldots, \varepsilon)$. From this we can derive the next result.

Proposition 1. Let $\mu$ be a Reinhardt measure on $\mathbf{C}^{n}$. Then $M_{z}$ is (jointly) bounded below, i.e., there exists $\delta>0$ such that $\left\|z_{1} f\right\|^{2}+$ $\cdots+\left\|z_{n} f\right\|^{2} \geq \delta^{2}\|f\|^{2}$ for all $f \in P^{2}(\mu)$.

Since $M_{z}$ is bounded below, we can use the groupoid machinery introduced in [CM] to analyze $C^{*}\left(M_{z}\right)$. This is done as follows. First, observe that $M_{z}$ is unitarily equivalent to an $n$-tuple of $n$-variable weighted shifts; for, if we let $e_{\alpha}:=z^{\alpha} /\left\|z^{\alpha}\right\|_{L^{2}(\mu)}(\alpha \in$ $\mathbf{Z}_{+}^{n}$ ), it follows from the Reinhardtness of $\mu$ that $\left\{e_{\alpha}\right\}_{\alpha \in \mathbf{Z}_{+}^{n}}$ is an orthonormal basis for $P^{2}(\mu)$, and that $M_{z_{i}} e_{\alpha}=w_{i}(\alpha) e_{\alpha}$, where

$$
\begin{gathered}
w_{i}(\alpha):=\left\|z^{\alpha+\varepsilon_{i}}\right\| /\left\|z^{\alpha}\right\|, \alpha \in \mathbf{Z}_{+}^{n}, i=1, \ldots, n, \\
\varepsilon_{i}:=(0, \ldots, 0, \stackrel{i}{1}, 0, \ldots, 0) .
\end{gathered}
$$

Similarly, if $\beta \in \mathbf{Z}_{+}^{n}$, the powers $M_{z}^{\beta}:=M_{z_{1}}^{\beta_{1}} \cdots M_{z_{n}}^{\beta_{n}}$ are associated with weight sequences $w_{\beta}(\cdot)$. Extend $w_{\beta}$ to all of $\mathbf{Z}^{n}$ via $w_{\beta}(\alpha):=0\left(\alpha \notin \mathbf{Z}_{+}^{n}\right)$, and let $\mathscr{A}$ be the closed translationinvariant subalgebra of $l^{\infty}\left(\mathbf{Z}^{n}\right)$ generated by $\left\{w_{\beta}\right\}_{\beta \in \mathbf{Z}_{+}^{n}}$, not including the constants. The maximal ideal space of $\mathscr{A}^{+}$, denoted $Y$, is a noncompact, locally compact Hausdorff space on which $\mathbf{Z}^{n}$ acts by translation. The map $\varphi: \mathbf{Z}^{n} \rightarrow Y$ given by $\varphi(\alpha)(a):=$ $a(\alpha), \alpha \in \mathbf{Z}^{n}, a \in Y$, is injective and open, and $X:=\varphi\left(\mathbf{Z}_{+}^{n}\right) \subseteq Y$ is compact. Thus, $X$ is a suitable compactification of $\mathbf{Z}_{+}^{n^{+}}[\mathrm{CM}$, 
Lemma 2.1 and Lemma 2.3]. If we let $\mathfrak{G}:=Y \times\left.\mathbf{Z}^{n}\right|_{X}:=\{(y, \alpha) \in$ $Y \times \mathbf{Z}^{n}: y \in X$ and $\left.y+\alpha \in X\right\}$, we see that $\mathfrak{G}$ is the groupoid obtained by reducing the transformation group $Y \times \mathbf{Z}^{n}$ to $X$, which therefore becomes the unit space of $\mathfrak{G}$. A careful analysis of $X$ leads to a detailed description of the ideal structure of $C^{*}\left(M_{z}\right)$, based on the correspondence between open invariant subsets of $X$ and closed ideals in $C^{*}\left(M_{z}\right)$. Since $X$ is obtained from $\mathbf{Z}_{+}^{n}$ by adding suitable limit points at infinity, we need to impose conditions on $\mu$ that guarantee a tractable identification of $X \backslash \mathbf{Z}_{+}^{n}$.

We shall say that a Reinhardt measure $\mu$ has convergent weight sequences if for every $i, j=1, \ldots, n$ and for every $\alpha \in \mathbf{Z}_{+}^{n}$, the sequence $\left\{w_{i}\left(\alpha+k \varepsilon_{j}\right)\right\}_{k=1}^{\infty}$ is convergent. The following theorem says that one can always assume that $\mu$ has no mass near the origin.

Theorem 2. Let $\mu$ be a Reinhardt measure on $\mathbf{C}^{n}$, let $K:=\operatorname{supp} \mu$, assume that $\mu$ has convergent weight sequences, let $\Omega$ be a neighborhood of $\partial \widehat{K}$, and let $\nu:=\left.\mu\right|_{\Omega}$. Then $C^{*}\left(M_{z}^{(\mu)}\right)$ is $*$-isomorphic to $C^{*}\left(M_{z}^{(\nu)}\right)$. Moreover, $M_{z}^{(\mu)}$ is a compact perturbation of $M_{z}^{(\nu)}$ (when each is regarded as an $n$-tuple of $n$-variable weighted shifts on $\left.l^{2}\left(\mathbf{Z}_{+}^{n}\right)\right)$. In particular, $M_{z}^{(\mu)}$ and $M_{z}^{(\nu)}$ have identical spectral pictures.

Our description of the spectral picture of $M_{z}$ relies on some special properties of the Koszul complex for $M_{z}$ in case $n=2$. Recall that

$$
K\left(M_{z}\right): 0 \rightarrow P^{2}(\mu) \stackrel{D^{0}(\mu)}{\longrightarrow} P^{2}(\mu) \oplus P^{2}(\mu) \stackrel{D^{1}(\mu)}{\longrightarrow} P^{2}(\mu) \rightarrow 0,
$$

where

$$
D^{0}(\mu) f=z_{1} f \oplus z_{2} f
$$

and

$$
D^{1}(\mu)(f \oplus g)=-z_{2} f+z_{1} g\left(f, g \in P^{2}(\mu)\right) .
$$

It follows from Proposition 1 that $D^{0}(\mu)$ is bounded below, and a trivial calculation then shows that $K\left(M_{z}\right)$ is exact at the middle stage, so that, by Theorem 1 , index $\left(M_{z}^{(\mu)}\right)=1$ once we establish that 0 is in the Fredholm domain of $M_{z}$. In the sequel, we assume that $n=2$.

To analyze $X$, we proceed as in [CM]. $\varphi\left(\mathbf{Z}_{+}^{2}\right)$ is an open invariant subset of $X$, whose associated ideal in $C^{*}\left(M_{z}\right)$ is the ideal of compact operators; on the other hand, we let $\infty_{G}$ denote the subset of $X$ consisting of all limit points of sequences 
$\left\{\varphi\left(\mathbf{k}^{(j)}\right)\right\}_{j=1}^{\infty}$, where $k_{i}^{(j)} \rightarrow+\infty$ for $i=1,2$. Clearly $\infty_{G}$ is a closed invariant subset of $X$, and $\left.\mathfrak{G}\right|_{\infty_{G}}=\infty_{G} \times \mathbf{Z}^{2}$. When both $\varphi\left(\mathbf{Z}_{+}^{2}\right)$ and $\infty_{G}$ are removed from $X$, we are left with two disjoint subsets, $\infty_{N}$ and $\infty_{E}$, consisting of all points in $X$ obtained by taking limits along vertical and horizontal directions, respectively; e.g., $\infty_{N}:=\left\{x \in X: x=\lim _{j} \varphi\left(\mathbf{k}^{(j)}\right),\left\{k_{1}^{(j)}\right\}\right.$ is bounded and $\left.k_{2}^{(j)} \rightarrow+\infty\right\}$. In the spectral and algebraic descriptions of $M_{z}$, the key role is played by $\infty_{G}$, on which we now focus our attention. Given a direction $\vec{u} \in \mathbf{R}_{+}^{2}$ we let

$$
\begin{aligned}
\infty_{\vec{u}}:=\left\{x \in X: \exists\left\{\varphi\left(\mathbf{k}^{(j)}\right)\right\}_{j=1}^{\infty} \text { with } \varphi\left(\mathbf{k}^{(j)}\right) \stackrel{w^{*}}{\longrightarrow} x, \mathbf{k}^{(j)}\right. \\
\left.=p_{j} \vec{u}+q_{j} \vec{u}^{\perp}, \text { and } q_{j} / p_{j} \rightarrow 0\right\} .
\end{aligned}
$$

Clearly $X=\bigcup_{\vec{u} \in \mathbf{R}_{+}^{2}} \infty_{\vec{u}}$, although two directions may give rise to the same limit points, and different limit points may correspond to the same direction. Nevertheless, the sets $\infty_{\vec{u}}$ carry important information.

A Reinhardt measure $\mu$ on $\mathbf{C}^{n}$ is said to have convergent weight sequences in all directions (c.w.s.a.d.) if for every direction $\vec{u} \in \mathbf{R}_{+}^{2}$ and every sequence $\left\{\mathbf{k}^{(j)}=p_{j} \vec{u}+q_{j} \vec{u}^{\perp}\right\} \stackrel{w^{*}}{\longrightarrow} x \in \infty_{\vec{u}}$ with $q_{j} / p_{j} \rightarrow$ 0 , the convergence of $\left\{q_{j}\right\}$ to some $q \in \mathbf{R}$ implies the convergence of $\left.\left\{w_{i}\left(\mathbf{k}^{(j)}\right)\right)\right\}_{j=1}^{\infty}, i=1,2$. Volumetric Lebesgue measure on a complete pseudoconvex Reinhardt domain and surface measure on the boundary of such a domain are two canonical examples of such Reinhardt measures; additional examples are given by Reinhardt measures $\mu$ such that $\left.\operatorname{supp} \mu\right|_{\partial \widehat{K}}=K \cap \partial \widehat{K}$. Intuitively, a measure $\mu$ has c.w.s.a.d. if it admits "balayage" to the boundary. There are, however, measures which do not have c.w.s.a.d.

Following the notation in [SSU], we let $C$ be the closed convex hull of $\log (\tau(K \backslash V))$. Then $\partial C=\partial^{0} C \cup \partial^{1} C$ (the boundary of $C$ is the union of its 0 - and 1-dimensional faces).

Proposition 2. Let $\mu$ be a Reinhardt measure on $\mathbf{C}^{2}$, and assume that $\mu$ has c.w.s.a.d. Then $\infty_{G}$ can be identified with $\partial C \backslash\left(F_{v} \cup F_{h}\right)$, where $F_{v}$ and $F_{h}$ are the vertical and horizontal (open) faces of $\partial C$, if they exist.

Each oblique 1-dimensional face of $\partial C$ gives rise to a direction $\vec{u} \in \mathbf{R}_{+}^{2}$; if $\mu$ has c.w.s.a.d., the corresponding $\infty_{\vec{u}}$ is topologically equivalent to the two-point compactification of the real line, with 
the action of $\mathbf{Z}^{2}$ given by $t+\left(\alpha_{1}, \alpha_{2}\right)=t+\alpha_{1} u_{1}-\alpha_{2} u_{2}$. This puts into evidence the presence of a copy of an irrational rotation $C^{*}$-algebra when $u_{1} / u_{2} \notin \mathbf{Q}$, intrinsic to the proof of (iii) below.

Theorem 3. Let $\mu$ be a Reinhardt measure on $\mathbf{C}^{2}$, and assume that $\mu$ has c.w.s.a.d. Then

(i) $M_{z}-\lambda$ is bounded below if and only if

$$
\lambda \notin\left(\exp \left(\partial^{0} C \times \mathbf{T}^{2}\right)\right)^{-},
$$

(ii) $M_{z}-\lambda$ is invertible if and only if $\lambda \notin \widehat{K}$,

(iii) $M_{z}-\lambda$ is Fredholm if and only if $\lambda \notin \partial \widehat{K}$,

(v) $\operatorname{index}\left(M_{z}-\lambda\right)=\left(\begin{array}{cc}1 & \text { if } \lambda \in \operatorname{int} \widehat{K} \\ 0 & \text { if } \lambda \notin \widehat{K} \text {. }\end{array}\right.$

Theorem 3 should be compared with [SSU, Theorem 1.3], where $\mu$ is volumetric Lebesgue measure on a complete pseudoconvex Reinhardt domain. Unlike the sheaf-theoretical methods used in [CS], [P], and [SSU] for the Bergman space case (obviously not applicable in the case of a general measure), our proof uses J. Bunce's characterization of the left spectrum [B], results from multiparameter spectral theory, and a covering lemma for the spectrum of $C^{*}\left(M_{z}\right)$ to reduce the problem to the case when int $\widehat{K}$ is the $L$ shaped domain $\Omega_{\delta_{1}, \delta_{2}}:=\left\{\left(z_{1}, z_{2}\right) \in \mathbf{C}^{2}:\left(\left|z_{1}\right|<\delta_{1},\left|z_{2}\right|<1\right)\right.$ or $\left(\left|z_{1}\right|<1,\left|z_{2}\right|<\delta_{2}\right\}\left(0<\delta_{1}, \delta_{2}<1\right)$. For $\Omega_{\delta_{1}, \delta_{2}}$, we calculate $\sigma_{T e}\left(M_{z}\right)$ by explicitly exhibiting a pair of (1-variable) bilateral weighted shifts acting on $l^{2}(\Gamma)(\Gamma$ a subgroup of $\mathbf{R})$, obtained via a suitably built faithful representation of $C^{*}\left(M_{z}\right) / \mathscr{K}$ associated with the direction $\vec{u}=\left(-\log \delta_{2},-\log \delta_{1}\right)$. Our techniques also allow us to handle certain cases of Reinhardt measures which do not have c.w.s.a.d., e.g., the example studied in [S].

Details of this work will be forthcoming.

\section{REFERENCES}

[BC] C. Berger and L. Coburn, Wiener-Hopf operators on $U_{2}$, Integral Equations Operator Theory 2 (1979), 139-173.

[BCK] C. Berger, L. Coburn and A. Korányi, Operateurs de Wiener-Hopf sur les spheres de Lie, C. R. Acad. Sci. Paris. Sér. A 290 (1980), 989-991.

[BCZ] C. Berger, L. Coburn and K. Zhu, Function theory on Cartan domains and the Berezin-Toeplitz symbol calculus, Amer. J. Math. (to appear).

[BdeM] L. Boutet de Monvel, On the index of Toeplitz operators of several complex variables, Invent. Math. 50 (1979), 249-272. 
[B] J. Bunce, The joint spectrum of commuting nonnormal operators, Proc. Amer. Math. Soc. 29 (1971), 499-505.

[Cob] L. Coburn, Singular integral operators and Toeplitz operators on odd spheres, Indiana Univ. Math. J. 23 (1973), 433-439.

[Con] J. B. Conway, Subnormal operators, Pitman Publ. Co., London, 1981.

[Cu] R. Curto, Applications of several complex variables to multiparameter spectral theory, Surveys of Some Recent Results in Operator Theory, v. II (J. Conway and B. Morrel, eds.), Pitman Res. Notes in Math. Ser. 192, Longman Sci. and Techn., London, 1988, pp. 25-90.

[CM] R. Curto and P. Muhly, $C^{*}$-algebras of multiplication operators on Bergman spaces, J. Funct. Anal. 64 (1985), 315-329.

[CS] R. Curto and N. Salinas, Spectral properties of cyclic subnormal m-tuples, Amer. J. Math. 107 (1985), 113-138.

[DH] R. Douglas and R. Howe, On the $C^{*}$-algebra of Toeplitz operators on the quarter plane, Trans. Amer. Math. Soc. 158 (1971), 203-217.

[MR] P. Muhly and J. Renault, $C^{*}$-algebras of multivariable Wiener-Hopf operators, Trans. Amer. Math. Soc. 274 (1982), 1-44.

[P] M. Putinar, Spectral theory and sheaf theory. II, Math. Z. 192 (1986), 473490.

[Ra] I. Raeburn, On Toeplitz operators associated with strongly pseudoconvex domains, Studia Math. 63 (1978), 253-258.

[SSU] N. Salinas, A. Sheu and H. Upmeier, Toeplitz operators on pseudoconvex domains and foliation $C^{*}$-algebras, Ann. of Math. (2) 130 (1989), 531565.

[S] A. Sheu, How are the Toeplitz $C^{*}$-algebras of Reinhardt domains affected by taking pseudoconvex hulls, Proc. Sympos. Pure Math. vol. 51, part 2, Amer. Math. Soc., Providence, RI, 1990, pp. 323-332.

[U] H. Upmeier, Toeplitz $C^{*}$-algebras on bounded symmetric domains, Ann. of Math. (2) 119 (1984), 549-576.

[V] U. Venugopalkrishna, Fredholm operators associated with strongly pseudoconvex domains in $\mathbf{C}^{n}$, J. Funct. Anal. 9 (1972), 349-373.

Department of Mathematics, University of Iowa, Iowa City, Iowa 52242

E-mail address: blaaulpd@uiamvs.bitnet

Department of Mathematical Sciences, I.U.P.U.I. 1125 E. 38th St., BoX 647, INDIANAPOLIS, INDIANA 46223

E-mail address: itgv100@indycms.bitnet 
